\title{
Appropriateness and adherence to guidelines in perioperative antimicrobial prophylaxis in patients undergoing clean surgery: results from a Quality Control Circle intervention
}

\section{Jian Zou (D70336641@qq.com )}

The People's Hospital of Pengzhou

Jia-yun Zhong

The People's Hospital of Pengzhou

Yu Liu

The People's Hospital of Pengzhou

\section{Dan Huang}

The People's Hospital of Pengzhou

Yan-xin Liu

The People's Hospital of Pengzhou

Xi-wen Li

The People's Hospital of Pengzhou

Wen-jun Gong

The People's Hospital of Pengzhou

Jia Tao

The People's Hospital of Pengzhou

Article

Keywords:

Posted Date: March 28th, 2022

DOI: https://doi.org/10.21203/rs.3.rs-1348246/v2

License: (c) (i) This work is licensed under a Creative Commons Attribution 4.0 International License. Read Full License 


\section{Abstract}

The present study aimed to exam the effect of quality control circle (QCC) activities on promoting the appropriate implementation of preoperative antibiotic prophylaxis in clean surgery. A QCC activity team was established to grasp the current situation of PAP in clean surgery procedure, set target, formulated corresponding countermeasures and implement and review them in stages. The plan-do-check-act method was cyclically applied. The appropriateness of PAP before (July to December 2018) and after (April to September 2019) the implementation of QCC activities were evaluated based on relevant international and Chinese perioperative antimicrobial prophylaxis guidelines. The appropriate rate of PAP was significantly improved from $68.72 \%(n=406)$ before QCC to $93.7 \%(n=508)$ post QCC implementation $(P \otimes 0.01)$. A significant improvement $(P \otimes 0.05)$ was also determined for each category: selection (from 78.82 to $96.06 \%$ ), duration (from 90.15 to $96.46 \%$ ), indication (from 94.09 to $97.64 \%$ ), timing of first dose (from 96.55 to $99.21 \%$ ), antibiotic usage (from 96.8 to $99.41 \%$ ), re-dosing of antibiotic (from 96.55 to 99.21\%). The appropriate application of PAP is improved through QCC activities, providing a useful reference for the prevention of antimicrobial-resistant bacteria and the development of medical quality improvement activities.

\section{Introduction}

Antimicrobial resistance (AMR) proposes a global challenge for both patients and health-care system ${ }^{[1]}$, is currently high on the global political agenda ${ }^{[2]}$. Microorganisms resistance is the main causative elements of hospital acquired infections (HAIs), of which surgical site infections (SSIs) are the most reported and surveyed type of $\mathrm{HAl}$, and accounting for up to $20 \%$ of all $\mathrm{HAI}^{[3]}$. SSI is one of the most common and severe clinical complications after surgeries, often causes prolonged hospitalization and delayed postoperative therapy, and substantially increase the health-care costs ${ }^{[4,5]}$. Clean surgery is defined as uninfected operative wound without inflammation, respiratory, alimentary, or genital of uninfected urinary track not entered according to the modified National Research Council wound classification criteria ${ }^{[6,7]}$. Although perioperative antimicrobial prophylaxis (PAP) guidelines across a wide array of surgical procedures have been proposed ${ }^{[8,9]}$. Still, suboptional use of antimicrobial for surgical prophylaxis is a common phenomenon worldwide, such as without indication, inappropriate selection or dosing of antimicrobial agents, prolonged duration of antimicrobial agents, incorrect timing of prophylaxis, which may result in AMR and higher healthcare costs ${ }^{[10]}$.

Quality control circle (QCC) is a process management and problem-solving technique founded in 1962 by Dr. Kaoru Ishikawa of Japan, which is viewed as a powerful tool to solve the problems in work and improve the complex work flow by the joint efforts of all members composed of staff from the same or similar or complementary workplaces ${ }^{[11]}$. Until 2001, QCC activities was introduced to healthcare industry in China, and numerous studies have reveled that the application of QCC could effectively raise the quality of medical service and ensure the safety of patients ${ }^{[12-14]}$. In our present study, a QCC program was carried out to improve the rational application of surgical antimicrobial prophylaxis in clean surgery 
procedures, and the plan-do-check-act (PDCA) method was implemented for 12 months. This was the first time that QCC program was implemented for the promotion of rational application of surgical antimicrobial prophylaxis in our hospital, after 12 months of continuous improvement, satisfied results have been achieved, as reported below.

\section{Material And Methods}

This study was carried out in the People's Hospital of Pengzhou, a tertiary hospital with 1,106 beds, 356 doctors and 485 nurses in Chengdu, China. The medical records of a total of 1092 patients underwent clean surgical procedures from July to December 2018 (before QCC) in our hospital were manually reviewed. In total, 1142 patients subjected to clean surgical procedures from January to June 2019 were enrolled as the intervention group (after QCC).

A total of 8 members QCC team was established. The director of pharmaceutical department served as the head of QCC group, and the circle members includes surgeon, clinical pharmacist, nurses, anesthetist, clinical microbiologist, infection control practitioner. The circle members proposed the theme of the QCC was 'to improve the appropriate implementation rate of surgical antimicrobial prophylaxis following brainstorming and voting, and the 'Spark Circle' was selected as circle name, meant to convey the concept of 'A single spark can start a prairie fire'.

Gantt chart was adopted to draw up the activities and duration of the separate steps of the implementation plan according to the processes and the theme of QCC, the person in charge of each step was confirmed. It was expected to take 24 weeks to complete all procedures, and 1-2 circle members are designated to supervise and implement each step.

A total of 1092 patients who underwent clean surgery from July to December 2019 were investigated, of which 406 patients received prophylactic antibiotics, and there have 182 problems during perioperative period, and the overall appropriate rate of PAP was $68.72 \%$ (127/406). The appropriate rate of implementation was analyzed by a flow chart for improvement and a checklist for irrational administration. The leading deficient quality indicators of PAP identified were inappropriate selection of antibiotics, prolonged duration of prophylaxis and PAP without indication. Furthermore, the circle capacity was set at $80 \%$ according the circle members' activeness and group cooperation following brainstorming and discussing, according to the formula of the target value, the target value =current value $\mathbb{~ ( c u r r e n t ~ v a l u e x v a l u e ~ o f ~ f o c u s ~} \times$ ability of circle $)=68.72 \% \bigotimes(68.72 \% \times 84.7 \% \times 80 \%)=22.16 \%$, the target value was to improve the implementation rate by $22.16 \%$ so as to achieve the appropriate rate of PAP in $90.88 \%$.

The cause of the inappropriate administration of PAP were analyzed by QCC members following brainstorming. Fishbone diagram was performed to facilitate root cause analysis discussion from the perspectives of people, equipment, materials, management, environment (Fig 1). Meanwhile, a questionnaire of hospital survey among doctors and nurses was carried out to assess hospital staff's perceptions of PAP. According to the $80 / 20$ principle, Pareto chart was adapted to demonstrate the 
essential causes of the inappropriate implementation that need to be improved are fear of SSI, lack of professional training, inadequate supervision, lack of standardized process and insufficient assessment (Fig 2).

According to the three key problems that can be solved, the final improvement strategies were implemented following brainstorming based on the comprehensive evaluation of the feasibility, autonomy and ability of QCC members. The leader of the QCC summarizes and evaluates the various methods, and then analyzes and modifies the methods through quality control tools. The specific methods are as follows:

Training about the knowledge of PAP. Experts in the field of anti-infection were invited to interpret the guidelines related to PAP and provided professional training and sufficient assessment for front-line doctors and nurses. In addition, various forms of publicity and training were carried out among surgeons, nurses, standard-trained doctors and new physicians. Furthermore, a booklet documented the standard operating procedure of PAP for various clean procedures according to the established criteria was distributed to each surgeons.

Strengthen pre-supervision of the whole-process: Clinical pharmacists participate in the formulation of prescriptions and conduct a real-time supervision on the whole process of PAP. Clinical pharmacist should remind the surgeons to modify the prescription immediately when irrational antibiotics were prescribed. If the opinions of pharmacist with surgeons are inconsistent, the pharmacist should consider the doctor's appeal and suggestions, and check the rationality of the prescription again, revising the evaluation standard if necessary.

Strengthen the evaluation of the rationality of prescription: Data on the characteristics of the surgical patients were collected from the hospital information system, the rationality of the PAP were evaluated by clinical pharmacists according to the established criteria. Clinical pharmacists summarized and analyzed the data of the unreasonable application of antimicrobial during the perioperative period, and reported to the medical management department every week. Furthermore, in view of some common problems of inappropriate application of PAP, hierarchical training were conducted by clinical pharmacists for key departments to elevate the professional knowledge of medical staff.

The differences on the appropriate implementation rate of PAP before and after the QCC activity were compared after the implementation of the QCC activities. The evaluation indicators including the indication, choice, duration and usage of antibiotics administered during perioperative period.

Statistical analysis. Data were represented as means \pm SEM. Statistical analyses were performed by using the SPSS 20.0 software (SPSS Inc.; IL, USA). Statistical comparisons between two groups were conducted by using One-way analysis of variance (ANOVA) followed by Bonferroni post hoc test. Difference between groups were considered statistically significant when a value of $P<0.05$.

\section{Results}


The comparison of the appropriateness of the perioperative antimicrobial prophylaxis between before and after the QCC activity was drawn. As shown in Tab 1, A significant improvement was also determined for each category: selection (from 78.82 to $96.06 \%$ ), duration (from 90.15 to $96.46 \%$ ), indication (from 94.09 to $97.64 \%$ ), timing of first dose (from 96.55 to $99.21 \%$ ), antibiotic usage (from 96.8 to $99.41 \%$ ), redosing of antibiotic (from 96.55 to $99.21 \%$ ), all the differences above were statistically significant $(P]$ 0.05). The total rate of appropriate surgical prophylaxis was significantly improved from $68.72 \%$ $(279 / 406)$ to $93.7 \%(476 / 508)$ after QCC intervention ( $P \otimes 0.01)$. After the QCC activity, the setting goal was achieved. Target reach rate=(improvement value-current value) $/$ (target value-current value) $\times 100 \%=$ $(93.7 \%-68.72 \%) /(90.88 \%-68.72 \%)=112.73 \%$, progress rate= (improvement value-current value)/current value $\times 100 \%=36.35 \%$. Furthermore, the incidence of SSI, other surgical complications and antibioticrelated ADR between before and after QCC activity were no statistically significant ( $P \otimes 0.05)$ (Tab 2).

In this QCC activity, obvious improvements of circle members were founded in terms of self-confidence, responsibility, work enthusiasm, problem-solving capability, communication skills, teamwork and motivation and skills of quality control (Fig 3). The implementation of QCC project not only enhances the individual ability, but also enables the whole team to form a highly enthusiastic atmosphere of study and work.

After the continuous improvement of the QCC activity, a standardized operation flow for the appropriate implementation of surgical antimicrobial prophylaxis in clean incision operations was established.

\section{Discussion}

Antimicrobial misuse and overuse are leading causes in the emergence of antimicrobial-resistant bacteria ${ }^{[15]}$. In our present study, only $68.72 \%$ of PAP were deemed to be appropriate before QCC activity when compared the current clinical practice of PAP with guidelines for antimicrobial prophylaxis in surgery published by China Ministry of Health, the leading deficient quality indicators of PAP identified were inappropriate choice of broad spectrum antibiotics, unnecessarily prolonged duration of prophylaxis, along with no indication to implementation of PAP without any reason. While the QCC intervention has a significant impact on the appropriateness of PAP in patients undergoing clean surgery, the overall compliance rate to guidelines rose to $93.7 \%$.

Although the availability of consensus guidelines for antibiotic prophylaxis in surgery clearly demonstrated the PAP indication, antibiotics selection, timing of first dose, antibiotic usage, duration of prophylaxis and re-dosing of antibiotic and all the parameters for clean surgical operations ${ }^{[9]}$. However, significant discrepancies between clinical practice of PAP and the guidelines in clean surgical procedures were founded before QCC in our hospital. Previous studies reported that lack of sufficient and effective education among surgeons seems to be the main barrier to adherence with PAP guidelines ${ }^{[16]}$, surgeons prescribe antibiotics according to their own principle that they trained in a wrong way in the past and it was counterintuitive for them to accept the new clinical practice guidelines about PAP ${ }^{[16]}$. Surgeons were accustomed to prescribe antibiotic prophylaxis and prolonged use of antibiotics in cases, as they falsely 
believed that maintaining antibiotics in the bloodstream of a post-operative patient was an effective precaution against $S S I S^{[17]}$. Thus, testing the compliance and acceptance of clinical guidelines among surgeons is of great significance for their effective implementation.

One of the most crucial issues for appropriate PAP depending on possible contaminating bacteria and their susceptibility to antimicrobials, whether antibiotics can reach an effective concentration at the surgical site ${ }^{[18]}$. In accordance with the international and native guidelines, first- or second- generation injectable cephalosporins was appropriately seleted and administered than other classes of antibiotics, cefazolin and cefuroxime were the first antibiotics of choice for majority of the surgical procedures according to the guidelines composed by China Ministry of Health. In our study, although the most frequently prescribed antimicrobials were cefazolin and cefuroxime, antibiotic selection was found to be inappropriate in $21.18 \%$ of cases before QCC intervention. Lower rates of appropriateness were demonstrated where broad-spectrum antibiotics such as third-generation cephalosporins, piperacillin/tazobartam, etc. were prescribed, as they are associated with increased emerge of extendedspectrum $\beta$-lactamases and Clostridioides difficile strians. The selection of appropriate antimicrobial agents in the present study pre QCC intervention (78.82\%) was higher to that of a recent study (55.3\%) ${ }^{[19]}$, but lower to that reported by Pittalis et al $(84.5 \%)^{[20]}$. While after the QCC intervention almost $96 \%$ received the appropriate antimicrobial agents for their surgical procedure.

Current antimicrobial prophylaxis in surgery guidelines recommend that the duration of PAP should not beyond $24 \mathrm{~h}$ after incision for clean procedures ${ }^{[21]}$. However, in almost $10 \%$ of the procedures in our study, the duration of PAP exceeded this time limit. Previous studies demonstrated that prolonged PAP can alter the institutional antibiogram and an individual's microbiome, which can lead to the emergence of bacterial resistance and may increase the incidence of antibiotic-associated complications ${ }^{[22,23]}$. Other studies revealed that a single intravenous dose or within $24 \mathrm{~h}$ administration of PAP is enough to prevent postoprerative infection in clean operations ${ }^{[23]}$. In line with prior studies, our results showed that shorter antimicrobial prophylaxis after QCC intervention has no effect on the incidence of SSI, other surgical complications and antibiotic-related ADR.

According to the international and native guidelines, some clean surgeries (such as breast and thyroid surgery, inguinal without a mesh, vascular vessel angiography, physical ablation of the tumor), antimicrobial prophylaxis was not recommended. However, in almost $6 \%$ of the procedures have no indication to use antimicrobial prophylaxis in our study. Antimicrobial overuse is the leading cause of AMR, and more than $30 \%$ of prescriptions are unnecessary according to the estimates of the US Centers for Disease Control and Prevention ${ }^{[24]}$. While after the QCC intervention, $99 \%$ of the antimicrobial prophylaxis have indication. Our finding highlight the importance of limiting antibiotics use in PAP.

In conclusion, the QCC activity has achieved good results in promoting the appropriateness of PAP in patients undergoing clean surgery. The principal finding of this study is that QCC implementation is associated with increased surgeon's perceptions of PAP. Moreover, QCC activities could effectively 
improve the self-confidence, problem-solving capability and skills of quality control of medical staff, and deserved application and promotion in medical quality improvement activities.

\section{Declarations}

\section{Funding}

This work was supported by the grants from Chengdu Municipal Health Commission [grant number 2020106].

\section{Author contributions}

Jian Zou and Jia-yun Zhong conceived the study. Yu Liu and Dan Huang performed data collection and analysis. Yan-xin Liu, Xi-wen Li, Wen-jun Gong and Jia Tao conduct the QCC activities. Jian Zou prepared the manuscript. All authors reviewed and edited the manuscript.

\section{Competing interests}

The authors declare that they have no competing interests

\section{Availability of data and materials}

Datasets used and/or analysed during the present study are available from the corresponding author on reasonable request.

\section{References}

1. Rawson TM, Wilson RC, O'Hare D, et al. Optimizing antimicrobial use: challenges, advances and opportunities. Nat Rev Microbiol. 19: 747-758 (2021).

2. Glover RE, Knight GM, Chandler CIR. Antimicrobial resistance at the G7. BMJ. 373: $\mathrm{n} 1417$ (2021).

3. Segala FV, Murri R, Taddei E, et al. Antibiotic appropriateness and adherence to local guidelines in perioperative prophylaxis: results from an antimicrobial stewardship intervention. Antimicrob Resist Infect Control. 9: 164 (2020).

4. Berrios-Torres SI, Umscheid CA, Bratzler DW, et al. Centers for Disease Control and Prevention Guideline for the Prevention of Surgical Site Infection, 2017. JAMA Surg. 152: 784-791 (2017).

5. Dior UP, Kathurusinghe S, Cheng C, et al. Effect of Surgical Skin Antisepsis on Surgical Site Infections in Patients Undergoing Gynecological Laparoscopic Surgery: A Double-Blind Randomized Clinical Trial. JAMA Surg. 155: 807-815 (2020).

6. Bratzler DW, Dellinger EP, Olsen KM, et al. Clinical practice guidelines for antimicrobial prophylaxis in surgery. Surg Infect (Larchmt). 14: 73-156 (2013).

7. ASHP Therapeutic Guidelines on Antimicrobial Prophylaxis in Surgery. American Society of HealthSystem Pharmacists. Am J Health Syst Pharm. 56: 1839-1888 (1999). 
8. Decker BK, Nagrebetsky A, Lipsett PA, et al. Controversies in Perioperative Antimicrobial Prophylaxis. Anesthesiology. 132: 586-597 (2020).

9. China NHaFPCo. Principles of clinical use of antibiotics [in Chines]. 2015. http://www.gov.cn/xinwe n/2015-08/27/content_29207 99.htm. Accessed 17 May 2018.

10. Muliani N, Herawati F, Yulia R, et al. Quantity and quality profiles of antibiotics pre, on, and post surgery in a hospital setting. Int J Clin Pharm. (2021).

11. Munchus G, 3rd. Employer-employee based quality circles in Japan: human resource policy implications for American firms. Acad Manage Rev. 8: 255-261 (1983).

12. Chen $P$, Yuan $T$, Sun $Q$, et al. Role of quality control circle in sustained improvement of hand hygiene compliance: an observational study in a stomatology hospital in Shandong, China. Antimicrob Resist Infect Control. 5: 54 (2016).

13. Liu Y, Lin B. Application of quality control circle in the treatment of moderate cancer pain in inpatients. Jpn J Clin Oncol. 50: 581-585 (2020).

14. Zhong $X$, Xiao LH, Wang DL, et al. Impact of a quality control circle on the incidence of catheterassociated urinary tract infection: An interrupted time series analysis. Am J Infect Control. 48: 11841188 (2020).

15. Khaw C, Oberle AD, Lund BC, et al. Assessment of Guideline Discordance With Antimicrobial Prophylaxis Best Practices for Common Urologic Procedures. JAMA Netw Open. 1: e186248 (2018).

16. Dimopoulou A, Kourlaba G, Psarris A, et al. Perioperative antimicrobial prophylaxis in pediatric patients in Greece: Compliance with guidelines and impact of an educational intervention. $J$ Pediatr Surg. 51: 1307-1311 (2016).

17. Tourmousoglou CE, Yiannakopoulou E, Kalapothaki V, et al. Adherence to guidelines for antibiotic prophylaxis in general surgery: a critical appraisal. The Journal of antimicrobial chemotherapy. 61: 214-218 (2008).

18. Gyssens IC. Preventing postoperative infections: current treatment recommendations. Drugs. 57: 175185 (1999).

19. Alshehhi HS, Ali AA, Jawhar DS, et al. Assessment of implementation of antibiotic stewardship program in surgical prophylaxis at a secondary care hospital in Ras Al Khaimah, United Arab Emirates. Sci Rep. 11: 1042 (2021).

20. Pittalis S, Ferraro F, Piselli P, et al. Appropriateness of surgical antimicrobial prophylaxis in the Latium region of Italy, 2008: a multicenter study. Surgical infections. 14: 381-384 (2013).

21. Bratzler DW, Dellinger EP, Olsen KM, et al. Clinical practice guidelines for antimicrobial prophylaxis in surgery. American journal of health-system pharmacy : AJHP : official journal of the American Society of Health-System Pharmacists. 70: 195-283 (2013).

22. Miranda D, Mermel LA, Dellinger EP. Perioperative Antibiotic Prophylaxis: Surgeons as Antimicrobial Stewards. J Am Coll Surg. 231: 766-768 (2020). 
23. Zhang W, Meng H, Mao C, et al. Utilization of neurosurgical perioperative antimicrobial prophylaxis in a Chinese teaching hospital. International journal of clinical pharmacy. 43: 1191-1197 (2021).

24. Fleming-Dutra KE, Hersh AL, Shapiro DJ, et al. Prevalence of Inappropriate Antibiotic Prescriptions Among US Ambulatory Care Visits, 2010-2011. JAMA. 315: 1864-1873 (2016).

\section{Tables}

Table 1. Comparison appropriateness of PAP before and after QCC activity

\begin{tabular}{|c|c|c|c|c|c|c|}
\hline \multirow{3}{*}{ Variables } & \multirow{2}{*}{\multicolumn{2}{|c|}{ Before QCC $₫ n=406 \rrbracket$}} & \multicolumn{4}{|c|}{ After QCC } \\
\hline & & & \multicolumn{2}{|c|}{$\square n=508 \square$} & \multirow[t]{2}{*}{$c^{2}$} & \multirow[t]{2}{*}{$P$} \\
\hline & $N$ & $\%$ & $N$ & $\%$ & & \\
\hline \multicolumn{7}{|l|}{ Antibiotic selection } \\
\hline 1.1 Appropriate implementation & 320 & $78.82 \%$ & 488 & $96.06 \%$ & \multirow[t]{2}{*}{9.324} & \multirow[t]{2}{*}{0.005} \\
\hline 1.2 Inappropriate implementation & 86 & $21.18 \%$ & 20 & $3.94 \%$ & & \\
\hline \multicolumn{7}{|l|}{ Duration of prophylaxis } \\
\hline 2.1 Appropriate implementation & 366 & $90.15 \%$ & 490 & $96.46 \%$ & \multirow[t]{2}{*}{6.417} & \multirow[t]{2}{*}{0.011} \\
\hline 2.2 Inappropriate implementation & 40 & $9.85 \%$ & 18 & $3.54 \%$ & & \\
\hline \multicolumn{7}{|l|}{ PAP indication } \\
\hline 3.1 Appropriate implementation & 382 & $94.09 \%$ & 496 & $97.64 \%$ & \multirow[t]{2}{*}{5.161} & \multirow[t]{2}{*}{0.019} \\
\hline 3.2 Inappropriate implementation & 24 & $5.91 \%$ & 12 & $2.36 \%$ & & \\
\hline \multicolumn{7}{|l|}{ Timing of first dose } \\
\hline 4.1 Appropriate implementation & 392 & $96.55 \%$ & 504 & $99.21 \%$ & \multirow[t]{2}{*}{7.354} & \multirow[t]{2}{*}{0.023} \\
\hline 4.2 Inappropriate implementation & 14 & $3.45 \%$ & 4 & $0.79 \%$ & & \\
\hline \multicolumn{7}{|l|}{ Antibiotic usage } \\
\hline 5.1 Appropriate implementation & 393 & $96.8 \%$ & 505 & $99.41 \%$ & \multirow[t]{2}{*}{6.983} & \multirow[t]{2}{*}{0.028} \\
\hline 5.2 Inappropriate implementation & 13 & $3.20 \%$ & 3 & $0.59 \%$ & & \\
\hline \multicolumn{7}{|l|}{ Re-dosing of antibiotic } \\
\hline 6.1 Appropriate implementation & 401 & $98.77 \%$ & 507 & $99.8 \%$ & \multirow[t]{2}{*}{7.025} & \multirow[t]{2}{*}{0.039} \\
\hline 6.2 Inappropriate implementation & 5 & $1.23 \%$ & 1 & $0.20 \%$ & & \\
\hline
\end{tabular}


Table 2. Comparison of surgical complications and antibiotic-related ADR before and after QCC intervention

\begin{tabular}{|llll|}
\hline Variables & $\begin{array}{l}\text { Before } \\
\text { QCC }\end{array}$ & $\begin{array}{l}\text { After } \\
\text { QCC }\end{array}$ & $P$ Value \\
\hline SSI & 2 & 1 & 0.289 \\
\hline Other surgical complications & & & \\
\hline Partial skin necrosis & 1 & 0 & 0.368 \\
\hline Hematoma or seroma & 6 & 4 & 0.542 \\
\hline Wound dehiscence & 3 & 2 & 0.435 \\
\hline $\begin{array}{l}\text { Antibiotic-related ADR } \\
\text { Allergic reaction(skin Rash, itching, flushing, macules, urticaria) }\end{array}$ & 7 & 6 & 0.586 \\
\hline $\begin{array}{l}\text { Digestive system(nausea, vomiting, acid reflux, abdominal } \\
\text { discomfort) }\end{array}$ & 6 & 4 & 0.542 \\
\hline $\begin{array}{l}\text { Cardiovascular system(phlebitis, palpitations, induration of } \\
\text { infusion site) }\end{array}$ & 0 & 0 & - \\
\hline \begin{tabular}{l} 
Nervous system(headache, convulsions, dizziness, quadriplegia) \\
\hline
\end{tabular} & 1 & 0 & 0.356 \\
\hline
\end{tabular}

\section{Figures}




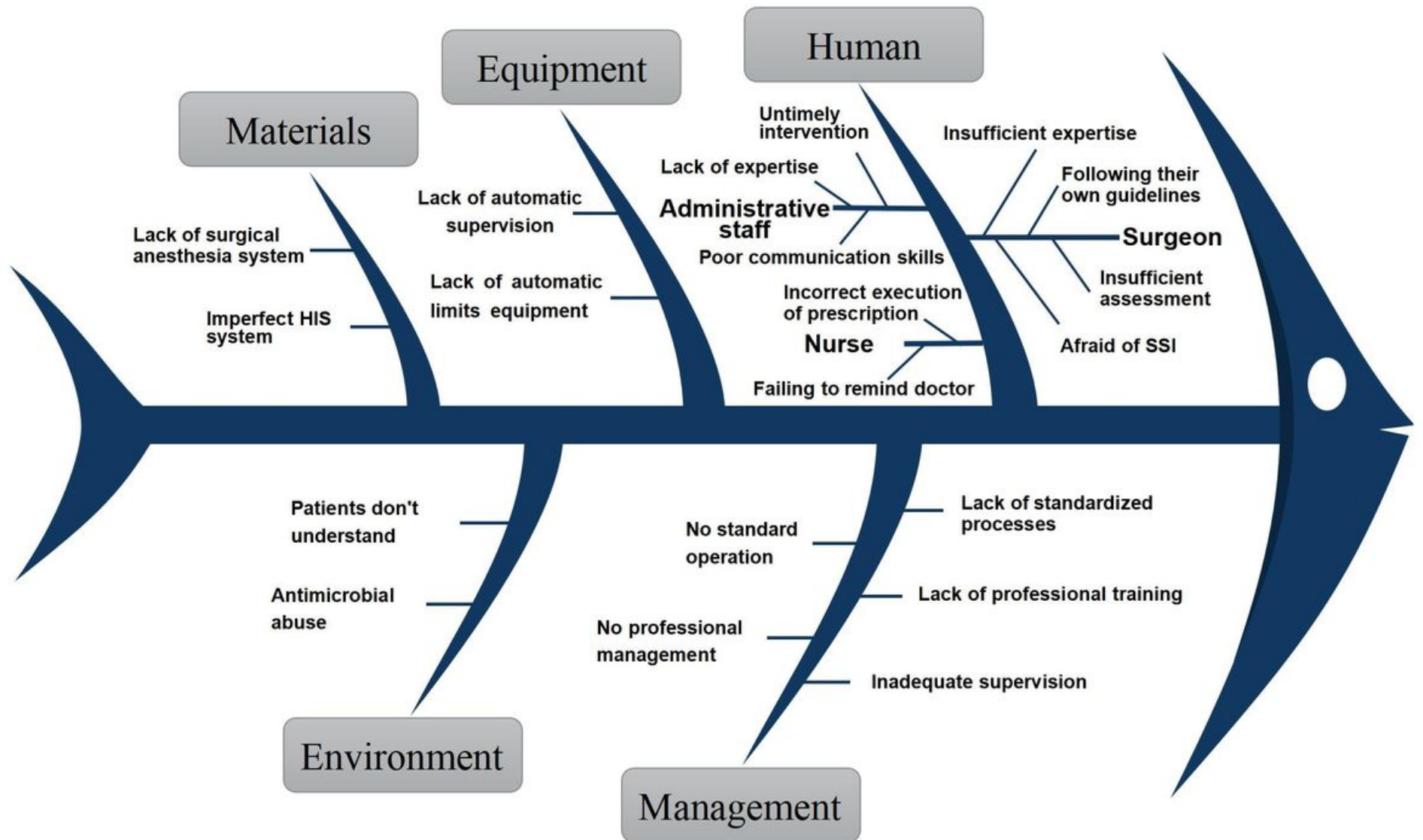

\section{Figure 1}

Analysis of the root causes of inappropriate implementation of PAP in clean surgery by Fishbone diagram 


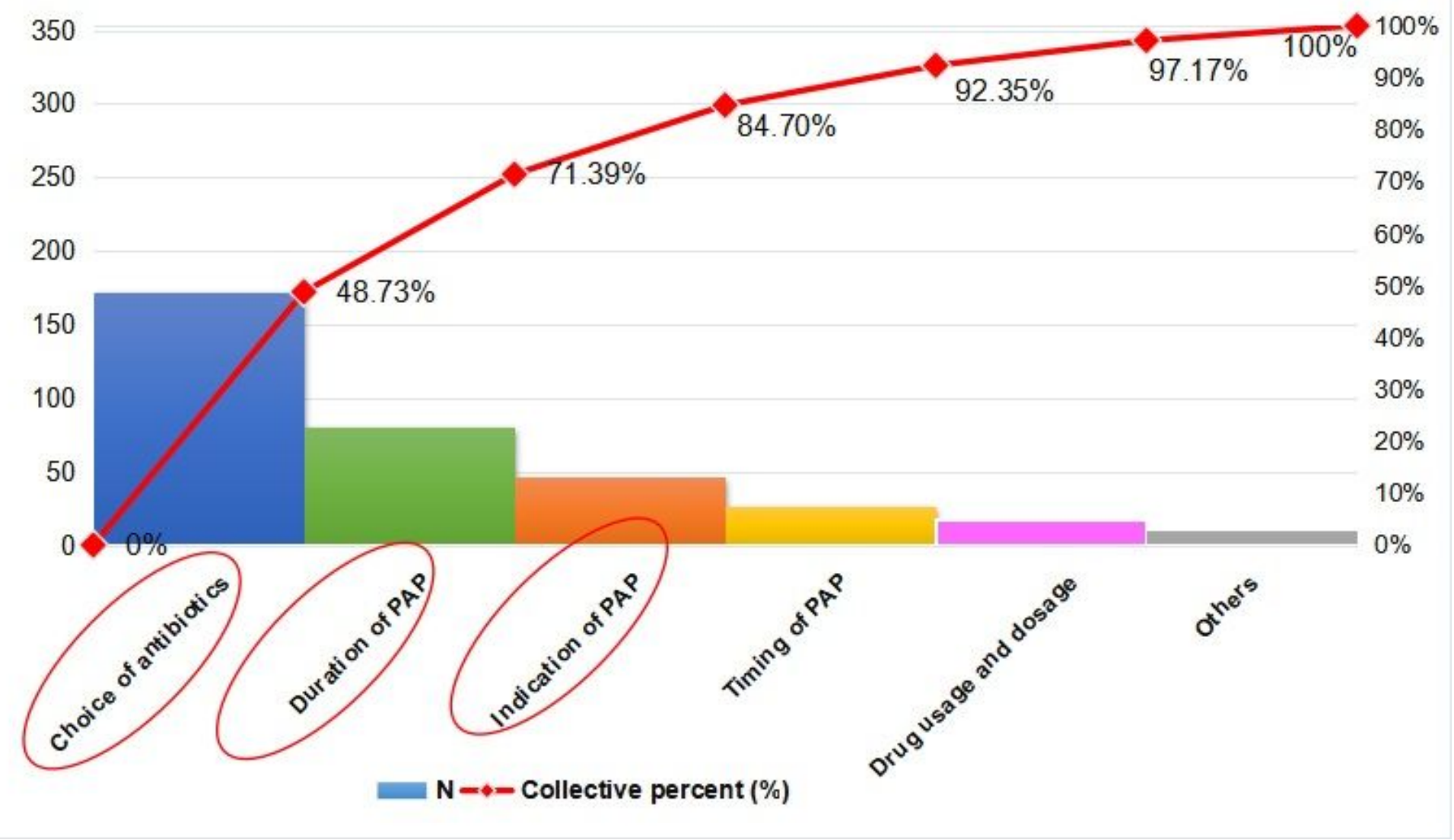

Figure 2

Pareto chart demonstrates the essential causes of the inappropriate implementation of PAP in clean surgery that need to be improved 
$\cdots$ Before QCC $\cdots$ After QCC

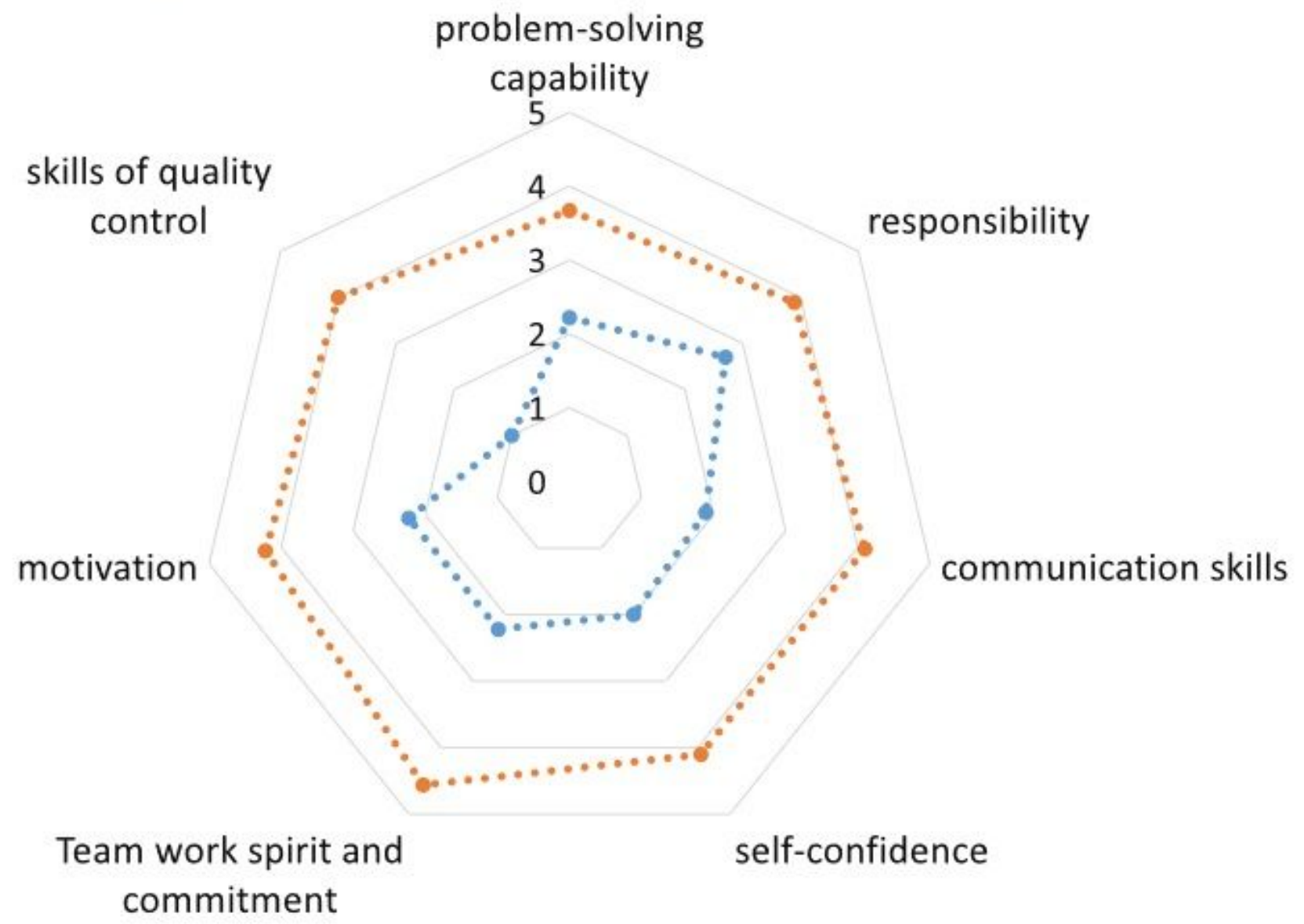

Figure 3

Analysis of the circle capability by radar diagram 